\title{
Clinical validation of immunoglobulin A nephropathy diagnosis in Swedish biopsy registers
}

This article was published in the following Dove Press journal:

Clinical Epidemiology

31 January 2017

Number of times this article has been viewed

\author{
Simon Jarrick ${ }^{1,2}$ \\ Sigrid Lundberg 3,4 \\ Adina Welander ${ }^{5,6}$ \\ C Michael Fored ${ }^{6}$ \\ Jonas F Ludvigsson ${ }^{2,7,8}$ \\ 'Department of Pediatrics, \\ Faculty of Health and Medicine, \\ Örebro University, ${ }^{2}$ Department \\ of Pediatrics, Örebro University \\ Hospital, Örebro, ${ }^{3}$ Department of \\ Nephrology, Karolinska University \\ Hospital, ${ }^{4}$ Department of Clinical \\ Science, Intervention and \\ Technology, Karolinska Institutet, \\ ${ }^{5}$ Boston Consulting Group, ${ }^{6} \mathrm{Clinical}$ \\ Epidemiology Unit, Department of \\ Medicine, ${ }^{7}$ Department of Medical \\ Epidemiology and Biostatistics, \\ Karolinska Institutet, Stockholm, \\ Sweden; ${ }^{8}$ Division of Epidemiology \\ and Public Health, School of \\ Medicine, University of Nottingham, \\ Nottingham, UK
}

Aims: The aims of this study were to validate the diagnosis of $\operatorname{Ig} A$ nephropathy $(\operatorname{IgAN})$ in Swedish biopsy registers against patient charts and to describe the clinical characteristics of patients with a biopsy indicating IgAN.

Methods: This is a population-based cohort study. Out of 4,069 individuals with a renal biopsy consistent with IgAN (biopsies performed in 1974-2011), this study reviewed patient charts of a random subset of 127 individuals. Clinical and biopsy characteristics at the time of biopsy were evaluated, and positive predictive values (PPV) were calculated with $95 \%$ confidence intervals (CI).

Results: Out of 127 individuals with a renal biopsy consistent with IgAN, 121 had a likely or confirmed clinical diagnosis of IgAN, primary or secondary to Henoch-Schönlein purpura, yielding a PPV of $95 \%(95 \% \mathrm{CI}=92 \%-99 \%)$. The median age at biopsy was 39 years (range: 4-79 years); seven patients $(6 \%)$ were $<16$ years. The male to female ratio was $2.8: 1$. The most common causes for consultation were macroscopic hematuria $(n=37 ; 29 \%)$, screening $(n=33$; $26 \%)$, and purpura $(n=14,11 \%)$. In patients with available data, the median creatinine level was $104 \mu \mathrm{mol} / \mathrm{L}$ (range $26-986 \mu \mathrm{mol} / \mathrm{L}, \mathrm{n}=110$ ) and glomerular filtration rate $75 \mathrm{~mL} / \mathrm{min} / 1.73 \mathrm{~m}^{2}$ (range $5-173 \mathrm{~mL} / \mathrm{min} / 1.73 \mathrm{~m}^{2}, \mathrm{n}=114$ ). Hypertension was noted in $59(46 \%)$ individuals. $\operatorname{IgA}$ deposits were reported in $97 \%$ of the biopsy records $(n=123)$, mesangial hypercellularity in $76 \%$ $(\mathrm{n}=96), \mathrm{C} 3$ deposits in $89 \%(\mathrm{n}=113)$, and $\mathrm{C} 1 \mathrm{q}$ deposits in $12 \%(\mathrm{n}=15)$.

Conclusion: A histologic diagnosis of IgAN has a high PPV for a diagnosis of IgAN confirmed by review of patient charts.

Keywords: general population-based, histopathology, IgA nephropathy, kidney, renal disease, validation studies

\section{Brief summary}

A cohort of 4,069 patients with IgA nephropathy (IgAN) from Swedish biopsy registers was collected for this study. The diagnosis was validated through review of patient charts from 127 randomly selected patients. The positive predictive value (PPV) of a biopsy consistent with $\operatorname{IgAN}$ was $95 \%$. Clinical characteristics of the cohort are in line with earlier studies.

\section{Introduction}

IgAN, which was first described by Berger and Hinglais in $1968,{ }^{1}$ is now recognized as the most frequent type of primary glomerulonephritis worldwide. ${ }^{2,3}$ Since subclinical disease is common and renal biopsy practice varies between countries, true incidence and prevalence data are difficult to establish. ${ }^{3}$ Incidence rates have been reported as $2-10$
Department of Pediatrics, Örebro University Hospital, 70I 85 Örebro, Sweden

Tel +46196023438

Fax +46 $191879 \quad 15$

Email simon.jarrick@gmail.com 
per 100,000 person-years ${ }^{3-8}$ with male to female ratios ranging from $1.3: 1$ to $6: 1 .^{4,7}$ IgAN is histologically characterized by IgA deposits in the glomerular mesangium. A renal biopsy with immunofluorescence staining or immunohistochemistry is required for the diagnosis. The archetypical clinical presentation of $\operatorname{IgAN}$ is that of a young adult with episodic macroscopic hematuria during an upper respiratory tract infection. Other patients are identified through asymptomatic urine abnormalities or through uremic symptoms late in the disease course. More seldom is there a clinical picture of nephrotic syndrome or acute renal failure. Some patients with a renal manifestation of IgAN also have systemic features of vasculitis in the skin (Henoch-Schönlein purpura [HSP]), intestine (gastrointestinal bleeding), or joints (arthritis), now classified as IgA vasculitis (IgAV). Histopathologically, there is no clear distinction between primary IgAN and IgAN related to HSP/IgAV, although the latter commonly shows more active inflammatory changes in the kidney. An important step in the pathogenesis of IgAN is the synthesis of high levels of poorly galactosylated IgA1 and IgG autoantibodies directed against GdIgA1, resulting in immune complex formation and deposition in the glomerular mesangium. Furthermore, this leads to mesangial cell proliferation, endocapillary proliferation, and varying degrees of segmental glomerulosclerosis and tubulointerstitial damage. ${ }^{9}$ Identification of mesangial IgA deposits by immunofluorescence or immunohistochemistry in renal biopsy is the key feature of the diagnosis. Such features are most often accompanied by proliferation of renal mesangial cells. Mesangial IgA deposits may rarely occur in other conditions, including human immunodeficiency virus (HIV) infection, chronic liver disease, and systemic lupus erythematosus (SLE).

The prognosis is variable, ranging from subclinical disease with isolated microscopic hematuria to severe renal disease progression. Up to $25 \%$ of affected individuals develop end-stage renal disease within 10-20 years from diagnosis. ${ }^{10,11}$ Recurrence in renal transplants occurs in $~ 30 \%$ of patients, with great discrepancy in different studies, mainly because of differences in follow-up times and in the threshold for performing a transplant biopsy. ${ }^{12}$

All renal biopsy specimens in Sweden are assessed in any of four pathology departments at the university hospitals in Stockholm, Göteborg, Linköping, and Malmö/Lund. Previously, data have been collected on a cohort consisting of all patients diagnosed with IgAN in Sweden, by performing computerized searches in all these four departments. ${ }^{13}$ This yielded a cohort of 4,069 unique individuals with a biopsy record of IgAN, diagnosed between 1974 and 2011. From all biopsies, data on 1) personal identity number, 2) date of biopsy,
3) topography (that the biopsy took place in the kidney), and 4) histologic diagnosis are available. This cohort could be an excellent source for further epidemiological studies. Since asymptomatic IgAN is common and renal biopsy practices vary between countries and regions, this cohort needs a thorough clinical description to increase external validity. As all registers may include imperfections due to ambiguous findings or simple typing errors, the IgAN diagnosis in this register-based cohort also requires internal validation.

The primary aim of this study was to validate the diagnosis of IgAN in Swedish biopsy registers against patient charts. A second aim was to describe the clinical characteristics of patients with a biopsy indicating IgAN.

\section{Subjects and methods Study design and primary study population}

The current study is a descriptive observational study of biopsy-verified IgAN in Sweden. The patient cohort consists of all individuals with a renal biopsy record of IgAN from 1974 to 2011, retrieved from pathology registers in the four units where all renal biopsies in Sweden are assessed. From this cohort, a random sample of 150 individuals were selected to validate the IgAN diagnosis and to characterize the patients. EpiTools (epitools.ausvet.com.au) was used and it was estimated that 139 patients need to be examined to detect a PPV of $90 \%$ with a $95 \%$ confidence interval (CI) of $85 \%-95 \%$. As patient charts in all the patients could not be expected, 150 patient charts were requested.

\section{Collection of renal biopsy data}

Renal biopsy with immunofluorescence staining or immunohistochemistry is required for the diagnosis of IgAN. In Sweden, evaluation of renal biopsies is centralized to four pathology departments (Stockholm, Göteborg, Malmö/Lund, and Linköping). Computerized records of biopsy reports are available in all of Sweden since the early 1990s; recording started in the 1980s, with some backward registration of older reports. Renal biopsy data were collected through computerized searches for relevant SnoMed (Systemized Nomenclature of Medicine - Clinical Terms ${ }^{14}$ ) codes for pathology diagnosis (IgAN, D67300) and topography (kidney, T71000). One region (Malmö/Lund) provided renal biopsy data but no SnoMed codes. Biopsy reports from this region were screened manually by a pathologist. ${ }^{13}$ For each biopsy, the study also obtained data on arrival date of the renal biopsy and personal identity number. ${ }^{15}$ Thus, a cohort of 4,069 unique individuals were identified with a biopsy report of IgAN. 


\section{Collection of clinical data and validation of IgAN diagnosis}

For the aforementioned 150 patients, the pathology departments provided information on the caregiver who had referred the patient for renal biopsy. Patients had been referred from 43 units in 32 different hospitals. Charts were received from $127(84.7 \%)$ patients and reviewed according to a standardized template. This included data on the cause of consultation, presenting symptoms, age, sex, comorbidities (including hepatitis and HIV infection), and symptoms or laboratory results that may suggest other diagnoses, like antineutrophil cytoplasmic antibody (ANCA) vasculitis or SLE. In ambiguous cases also, the clinical course during follow-up was evaluated to distinguish IgAN from differential diagnoses. Glomerular filtration rate (GFR) was described with Iohexol or ${ }^{51} \mathrm{Cr}$-ethylenediaminetetraacetic acid clearance data, when available, and otherwise estimated from serum creatinine levels, using the Modification of Diet in Renal Disease Study (MDRD) formula in adults $(\geq 16 \text { years })^{16}$ and the Schwartz bedside formula in children ( $<16$ years) ${ }^{17}$

Isolated $\operatorname{IgAN}$ and $\operatorname{IgAN}$ with extra-renal vascular manifestations (HSP/IgAV) were treated as one common entity. Patients were divided into either of three categories (Figure 1): confirmed IgAN, likely IgAN, or unlikely IgAN.

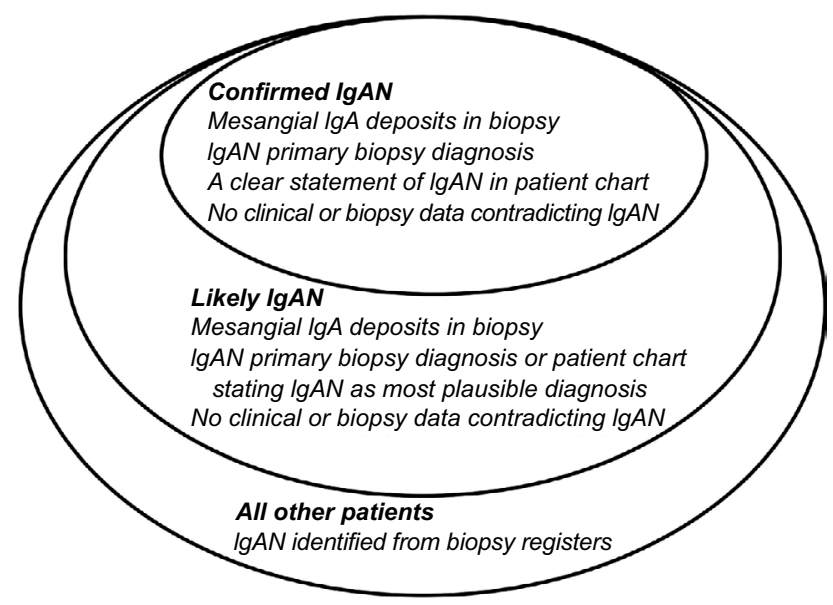

Figure I Definition of confirmed and likely IgAN. Abbreviation: $\lg \mathrm{AN}, \lg \mathrm{A}$ nephropathy.
A confirmed IgAN diagnosis required 1) a description of mesangial IgA deposits in biopsy report, 2) IgAN as primary biopsy diagnosis, 3) a clear statement of IgAN in the patient chart, and 4) no clinical or biopsy data contradicting IgAN. Likely IgAN required 1) a description of mesangial IgA deposits in biopsy report, 2) either IgAN as primary biopsy diagnosis or a statement in patient chart of IgAN as the most plausible diagnosis, and 3) no clinical or biopsy data contradicting IgAN. When these criteria were not met, the diagnosis was considered unlikely.

\section{Statistics}

The PPV was calculated with $95 \% \mathrm{CI}$ of having an IgAN+ biopsy, using confirmed and/or probable IgAN as the gold standard of diagnosis. Calculations were made using Microsoft Excel Program (Microsoft Corporation, Redmond, WA, USA).

\section{Compliance with ethical standards}

The current study was approved by the Stockholm Ethics Review Board (Jan 22, 2014; approval number: 2013/2095$31 / 2$ ). For this type of study, formal consent was deemed unnecessary according to the Stockholm Ethics Review Board.

\section{Results}

\section{Validation of IgAN diagnosis from biopsy reports}

Table 1 presents the characteristics of the 127 patients with patient chart data and 23 patients without such data. Two units (with a total of 10 patients) did not provide any patient charts. For another 13 patients, charts were either incomplete or could not be located. Out of the remaining 127 patients, IgA deposits were found to at least some degree in 123 patients (97\%), and IgAN was confirmed or likely in 121 patients (PPV $=95 \%$, 95\% CI =92\%-99\%), with a confirmed IgAN diagnosis in 116 patients ( $\mathrm{PPV}=91 \%, 95 \% \mathrm{CI}=86 \%-96 \%$ ). In three patients with confirmed IgAN, ANCAs were demonstrated in low titers; none of those showed clinical or histopathological features of ANCA-associated vasculitis,

Table I Characteristics of unique individuals at first IgAN+ renal biopsy (in I 27 of I50 randomly selected cases, patient charts were available; I 3 of them had a confirmed clinical diagnosis of IgAN)

\begin{tabular}{llll}
\hline & All individuals & Confirmed IgAN & Unavailable patient charts \\
\hline $\mathrm{n}$ & 127 & 116 & 23 \\
Age, years (median, range) & $39(4-79)$ & $39(4-79)$ & $27(9-69)$ \\
Children $<16$ years, $\mathrm{n}(\%)$ & $7(6)$ & $7(6)$ & $6(26)$ \\
Females, $\mathrm{n}(\%)$ & $33(26)$ & $30(26)$ & $5(22)$ \\
Year of first renal biopsy & $2001(1978-2010)$ & $2001(1978-2010)$ & $1991(1980-2010)$ \\
(median, upper range) & & &
\end{tabular}

Abbreviation: IgAN, IgA nephropathy. 
and ANCA was judged unspecific. In two patients with confirmed IgAN, there was evidence of a second renal diagnosis (ANCA-associated vasculitis and drug-induced interstitial nephritis, respectively). One patient had a confirmed chronic hepatitis $\mathrm{C}$ infection and demonstrated virus-like endothelial inclusions, indicating that IgAN might be secondary to hepatitis C. HSP-related IgAN/IgAV with extra-renal manifestations was present in 16 patients (13\%). In six patients, IgAN was ruled out or considered unlikely: in three patients where IgA deposits were not mentioned in the biopsy report, IgAN was ruled out as the primary renal diagnosis (one patient had SLE, and the other two had acute tubular necrosis due to Puumala virus); in three patients with non-IgAN-renal diagnoses, mesangial IgA and C3 deposits were considered incidental findings, although a concurrent IgAN could not be excluded (one patient with acute steroid responding nephrotic syndrome, where electronic microscopy showed widening of podocyte foot processes as in minimal change glomerulonephritis, one patient with acute renal failure, high titers of antibodies to glomerular basement membrane, and linear IgG fluorescence pattern as in Goodpasture's disease, and one patient with acute onset flank pain and macroscopic hematuria, where a toxic effect of ethylene glycol was considered a more likely explanation).

In five patients, IgAN was considered likely but could not be fully confirmed (failed immunofluorescence staining, $\mathrm{n}=1$; mainly capillary location of $\operatorname{IgA}$ deposits and incomplete patient chart, $\mathrm{n}=1$; chronic changes dominating and $\operatorname{IgAN}$ stated "probable" by both pathologist and clinician, $n=3$ ).

\section{Age and sex of individuals with an $\lg \mathrm{AN}+$ renal biopsy}

The male to female ratio in this study group was 2.8:1. The majority were adults at the time of first renal biopsy; $6 \%$ were children ( $<16$ years; Table 1$)$. Additional data are given in Table 1.

\section{Clinical characteristics of individuals with IgAN at the time of biopsy}

Clinical characteristics according to the 127 reviewed patient charts are presented in Table 2. Microscopic or macroscopic hematuria was recorded in 116 patients (91\%), and data of eight patients were missing. Proteinuria was measured with different methods. Significant proteinuria, defined as urinary albumin excretion of $\geq 1 \mathrm{~g} / 24 \mathrm{~h}$ or $\geq 1 \mathrm{~g} / \mathrm{L}$, or protein excretion of $\geq 1.5 \mathrm{~g} / \mathrm{L}$, or an urinary albumin to creatinine ratio of $\geq 100 \mathrm{~g} / \mathrm{mole}$, was present in 56 patients. Forty-two patients (33\%) had less severe or no proteinuria (data missing in 29
Table 2 Clinical characteristics and laboratory data at the time of first lgAN+ renal biopsy $(n=127)$

\begin{tabular}{|c|c|}
\hline Cause of consultation & n (\%) \\
\hline Infection-related macroscopic hematuria & $27(2 I)$ \\
\hline Macroscopic hematuria (without mention of infection) & $10(8)$ \\
\hline Purpura & $14(11)$ \\
\hline Malignant hypertension & $5(4)$ \\
\hline Nephrotic syndrome & $4(3)$ \\
\hline Renal failure & $5(4)$ \\
\hline Screening & $33(26)$ \\
\hline Joint pain & $3(2)$ \\
\hline Flank pain & $8(6)$ \\
\hline Other & $5(4)$ \\
\hline \multicolumn{2}{|l|}{ Family history } \\
\hline Family history of renal disease & $20(16)$ \\
\hline Family history of ESRD & $9(7)$ \\
\hline Family history of IgAN & $8(6)$ \\
\hline \multicolumn{2}{|l|}{ Laboratory data } \\
\hline Creatinine $(\mu \mathrm{mol} / \mathrm{L} ; \mathrm{n}=\mathrm{I} \mathrm{I} 0$; median [range] $)$ & $104(26-986)$ \\
\hline GFR $\left(\mathrm{mL} / \mathrm{min} / \mathrm{I} .73 \mathrm{~m}^{2} ; \mathrm{n}=\mid \mathrm{I} 4\right.$; median [range] $)$ & $75(5-173)$ \\
\hline CKD I-2 GFR $\geq 60$ (n; \%) & $75(59)$ \\
\hline CKD 3 GFR $\geq 30$ and $<60$ (n; \%) & $20(16)$ \\
\hline CKD 4 GFR <30 (n; \%) & $21(17)$ \\
\hline Systolic BP (mmHg; $n=102 ;$ mean \pm SD) & $138 \pm 22$ \\
\hline Diastolic BP (mmHg; $n=102 ;$ mean \pm SD) & $83 \pm 15$ \\
\hline $\begin{array}{l}\text { Hypertension }(\mathrm{BP}>\mid 40 / 80 \mathrm{mmHg}) \text { or on } \\
\text { antihypertensive treatment }(\mathrm{n} ; \%)\end{array}$ & $59(46)$ \\
\hline Antinuclear antibodies $(n=68)(n ; \%)$ & $6(5)$ \\
\hline Viral hepatitis $(n=5 I)(n ; \%)$ & $3(2)$ \\
\hline Antineutrophilic cytoplasmic antibodies (n; \%) & $5(4)$ \\
\hline Microscopic or macroscopic hematuria $(n=\mid 19)$ & $116(91)$ \\
\hline $\begin{array}{l}\text { Significant proteinuria (dU-albumin }>1 \mathrm{~g} / 24 \mathrm{~h} \text {, } \\
\mathrm{dU} \text {-protein }>1.5 \mathrm{~g} / 24 \mathrm{~h}, \mathrm{U} \text {-albumin }>\mathrm{g} / \mathrm{L} \text { or } \\
\text { U-albumin/creatinine ratio }>100 \mathrm{~g} / \mathrm{mole} \text { ) }(\mathrm{n}=98)\end{array}$ & $56(44)$ \\
\hline
\end{tabular}

Abbreviations: IgAN, IgA nephropathy; ESRD, end-stage renal disease; GFR, glomerular filtration rate; CKD, chronic kidney disease; BP, blood pressure; SD, standard deviation.

cases). In a majority of patients $(59 \%)$, renal function was well preserved at inclusion (GFR $\geq 60 \mathrm{~mL} / \mathrm{min} / 1.73 \mathrm{~m}^{2}$ ). In $17 \%$, there was a severe reduction in GFR $(<30 \mathrm{~mL} /$ $\min / 1.73 \mathrm{~m}^{2}$ ). Hypertension was noted in $46 \%$ of the patients, and $28 \%$ were on angiotensin converting enzyme inhibitor or angiotensin II receptor antagonist therapy at the time of renal biopsy. Approximately 16\% had a family history of renal disease: in eight cases $(6 \%)$, there was a family history of IgAN (Table 2).

\section{Biopsy findings}

Data from these biopsy reports mentioned IgA deposits of at least moderate fluorescence or abundance in $84 \%$ of the cases $(n=107)$, mesangial hypercellularity in $76 \%(n=96), C 3$ 
Table 3 Renal biopsy findings

\begin{tabular}{lll}
\hline Biopsy findings & All individuals & Confirmed IgAN \\
\hline $\mathrm{n}$ & 127 & 116 \\
IgA deposits: moderate or rich & $107(84)$ & $105(91)$ \\
amount or moderate or strong & & \\
fluorescence, $\mathrm{n}(\%)$ & & \\
Mesangial hypercellularity, $\mathrm{n}(\%)$ & $96(76)$ & $92(79)$ \\
C3 deposits, $\mathrm{n}(\%)$ & $\mathrm{II}(89)$ & $104(90)$ \\
Clq deposits, $\mathrm{n}(\%)$ & $15(12)$ & $14(12)$ \\
\hline
\end{tabular}

Abbreviation: IgAN, IgA nephropathy.

deposits in $89 \%(n=113)$, and C1q deposits in $12 \%(n=15)$ (Table 3). In 21 patients, a second renal biopsy had been performed: in 13 cases because of ambiguous findings in the first biopsy, and in the remaining cases of renal transplant to assess disease recurrence or transplant rejection.

\section{Patients with unavailable patient charts}

Age and sex distribution were similar in patients with and without available patient charts (Table 1). However, in patients without charts, the renal biopsy had been performed on average 10 years earlier than in patients with available clinical data (median year of biopsy 1991 vs 2001). The earlier year of biopsy in those with a missing patient chart may reflect the fact that patient charts are only stored for a certain period of time in Sweden.

\section{Discussion}

This paper describes the validation of the IgAN diagnosis according to renal biopsy reports in Sweden. In total, 4,069 unique individuals were identified with a biopsy indicating IgAN through computerized searches in pathology reports using SnoMed codes, supplemented by manual screening in one department not providing SnoMed codes. Since a renal biopsy is required for IgAN diagnosis, and renal biopsy evaluation is centralized to only four departments in Sweden, this cohort can be regarded as truly populationbased. Patient charts for a subset of 127 randomly selected individuals were examined for $\operatorname{IgAN}$ validation including thorough reevaluation of biopsy reports. In all, the results indicate that a biopsy report of IgAN from Swedish pathology departments is highly specific for true IgAN, with a PPV of 95\% for confirmed or likely IgAN.

The current patient chart review found that $\sim 19$ out of 20 patients with a biopsy report with IgAN had clinical IgAN. Among other diagnoses with IgAN-like biopsies were SLE and acute tubular necrosis due to Puumala virus infection. SLE patients sometimes have sparse immune depositions of IgA and C3 and only mild mesangial proliferation early in the disease course before developing the typical picture of "full house" immune depositions including also IgG and IgM. Clinical follow-up in general helps to differentiate between the diseases. On rare occasions, mesangial IgA deposits can also be found when capillary damage occurs, for example, in conditions characterized by acute tubular necrosis and gross hematuria.

A substantial proportion of patients (11\%) were stained positive for C1q. There is a debate of whether or not the presence of $\mathrm{Clq}$ in renal biopsy should rule out the diagnosis of $\operatorname{IgAN}$ or is a marker of more severe prognosis in this disease. ${ }^{18,19}$ SLE nephritis and C1q nephropathy are among the differential diagnoses here. The decision to classify these cases as confirmed or likely IgAN is based on the evaluation of the patient charts where no other clinical or laboratory signs of SLE were described. The predominance of IgA over $\mathrm{C} 1 \mathrm{q}$ staining makes the diagnosis of $\mathrm{C} 1 \mathrm{q}$ nephropathy less likely. Combining renal biopsy data with data from the National Patient Register ${ }^{20}$ in future studies should help rule out some cases with false-positive IgAN since at least SLE is often diagnosed in the Swedish National Patient Register.

The male to female ratio in this study (2.8:1) is consistent with earlier literature, ${ }^{4}$ and a vast majority of individuals were diagnosed in adulthood. Two retrospective Japanese studies ${ }^{6,7}$ based on school urinary dipstick screening programs show a less marked male predominance. Since IgAN is often asymptomatic and spontaneous recovery sometimes occurs, this might be an indication that female IgAN incidence is underestimated in studies that are not screening-based or could reflect a true difference in sexual predilection between genetically diverse populations. The present study shows relatively few cases of IgAN diagnosed in childhood, compared to older studies. This can in part be explained by the fact that one of the departments failing to provide patient charts cared for children. Dropouts included $(n=150), 9.3 \%$ of all individuals were diagnosed in childhood. In Sweden, renal biopsy is often postponed in children with suspected mild to moderate IgAN with normal renal function and minimal proteinuria, especially when nephropathy is associated with cutaneous vasculitis (HSP); therefore, this study may underestimate the true proportion of IgAN diagnosed during childhood.

The most common causes of referral for renal biopsy were macroscopic hematuria related to infectious disease, urinary abnormalities detected by screening, and purpura of the skin. These findings are comparable to those in other patient cohorts. ${ }^{11}$ One in six individuals in this validation study had a family history of renal disease, which is in line with one previous Swedish study. ${ }^{21}$

So far, most of the epidemiological studies on renal disease emanating from Sweden have used the Swedish National 
Patient Registry to ascertain the disease. While that register has a high PPV for many chronic disorders, ${ }^{20} 95 \%$ as seen for renal biopsy data must be regarded as very high. Also, the use of biopsy data to ascertain IgAN has a high sensitivity, since renal biopsy is a condition for diagnosis. Renal biopsy data also have the advantage that they enable researchers to examine the risk factors for IgAN and future complications for the same disease according to biopsy status.

Renal biopsy registers are an important source of research worldwide. ${ }^{2}$ In a study by the Spanish Society of Nephrology, renal biopsy data showed a biopsy rate of 4.8/100,000 inhabitants. The most common pathological finding in adults was that of IgAN. ${ }^{22}$ Renal biopsy data have been used with great success elsewhere not only to examine both geographical and socioeconomic differences in disease but also to improve patient care. Investigations based on the Scottish Renal Biopsy Register showed that both the incidence of renal biopsy and a diagnosis of IgAN were overrepresented in socially deprived areas, independent of demographic data, and there were some differences in the clinical picture between patients from different socioeconomic groups. ${ }^{8}$ In Norway, record linkage between the Kidney Biopsy Registry, the Norwegian Cause of Death Registry, and the Norwegian Renal Registry unraveled a twofold mortality risk for patients with IgAN compared to the general population. This increase of mortality was mainly seen after initiation of renal replacement therapy. ${ }^{23}$ A nationwide Kidney Biopsy Registry, linked to the well-established Swedish Renal Registry, has recently been started up in Sweden and will in the near future allow for a wide range of epidemiologic studies on all forms of glomerulonephritis and other renal diagnoses.

\section{Conclusion}

It is concluded that in Sweden, renal biopsies consistent with IgAN have a high predictive value for the diagnosis of IgAN confirmed by review of patient charts, and patient characteristics of individuals with biopsy-verified IgAN are similar to that reported from international literature.

\section{Acknowledgment}

This study was performed with grants from the research committee of Örebro County Council (grant number OLL-406471).

\section{Disclosure}

The authors report no conflicts of interest in this work.

\section{References}

1. Berger J, Hinglais N. Les Dépôts intercapillaires d'IgA-IgG [Intercapillary deposits of IgA-IgG]. J Urol Néphrologie. 1968;74(9):694-695. French.

2. Pesce F, Schena FP. Worldwide distribution of glomerular diseases: the role of renal biopsy registries. Nephrol Dial Transplant. 2010;25(2): 334-336.

3. McGrogan A, Franssen CFM, Vries CS de. The incidence of primary glomerulonephritis worldwide: a systematic review of the literature. Nephrol Dial Transplant. 2011;26(2):414-430.

4. Donadio JV, Grande JP. IgA nephropathy. NEngl J Med. 2002;347(10): 738-748.

5. Simon P, Ramee M-P, Boulahrouz R, et al. Epidemiologic data of primary glomerular diseases in western France. Kidney Int. 2004;66(3):905-908.

6. Utsunomiya Y, Koda T, Kado T, et al. Incidence of pediatric IgA nephropathy. Pediatr Nephrol. 2003;18(6):511-515.

7. Shibano T, Takagi N, Maekawa K, et al. Epidemiological survey and clinical investigation of pediatric IgA nephropathy. Clin Exp Nephrol. 2015; 20(1):111-117.

8. McQuarrie EP, Mackinnon B, McNeice V, Fox JG, Geddes CC. The incidence of biopsy-proven IgA nephropathy is associated with multiple socioeconomic deprivation. Kidney Int. 2014;85(1):198-203.

9. Boyd JK, Cheung CK, Molyneux K, Feehally J, Barratt J. An update on the pathogenesis and treatment of $\operatorname{IgA}$ nephropathy. Kidney Int. 2012;81(9):833-843.

10. D'Amico G. Natural history of idiopathic IgA nephropathy and factors predictive of disease outcome. Semin Nephrol. 2004;24(3):179-196.

11. Barratt J, Feehally J. Treatment of IgA nephropathy. Kidney Int. 2006; 69(11):1934-1938.

12. Ponticelli C, Glassock RJ. Posttransplant recurrence of primary glomerulonephritis. Clin J Am Soc Nephrol. 2010;5(12):2363-2372.

13. Welander A, Sundelin B, Fored M, Ludvigsson JF. Increased risk of IgA nephropathy among individuals with celiac disease. J Clin Gastroenterol. 2013;47(8):678-683.

14. SNOMED CT: The Global Language of Healthcare. Available from: http://www.ihtsdo.org/snomed-ct. Accessed December 6, 2016.

15. Ludvigsson JF, Otterblad-Olausson P, Pettersson BU, Ekbom A. The Swedish personal identity number: possibilities and pitfalls in healthcare and medical research. Eur J Epidemiol. 2009;24(11):659-667.

16. Levey AS, Coresh J, Greene T, et al. Using standardized serum creatinine values in the modification of diet in renal disease study equation for estimating glomerular filtration rate. Ann Intern Med. 2006;145(4): 247-254.

17. Schwartz GJ, Muñoz A, Schneider MF, Mak RH, Kaskel F, Warady BA, Furth SL. New equations to estimate GFR in children with CKD. $J$ Am Soc Nephrol. 2009;20(3):629-637.

18. Nasri H. Letter to the article: association of $\mathrm{C} 1 \mathrm{q}$ deposition with renal outcomes in IgA nephropathy Clin Nephrol. 2013;80:98-104. Clin Nephrol. 2014;81(03):228-229.

19. Lee H-J, Choi SY, Jeong KH, et al. Association of C1q deposition with renal outcomes in IgA nephropathy. Clin Nephrol. 2013;80(2):98-104.

20. Ludvigsson JF, Andersson E, Ekbom A, et al. External review and validation of the Swedish national inpatient register. BMC Public Health. 2011;11:450.

21. Lundberg S. Factors Predicting Progression of Chronic Kidney Disease in IgA Nephropathy. Inst för medicin, Solna / Dept of Medicine, Solna; 2012. Available from: http://openarchive.ki.se/xmlui/handle/10616/40955. Accessed October 30, 2015.

22. Rivera F, López-Gómez JM, Pérez-García R; Spanish Registry Glomerulonephritis. Frequency of renal pathology in Spain 1994-1999. Nephrol Dial Transplant. 2002;17(9):1594-1602.

23. Knoop T, Vikse BE, Svarstad E, et al. Mortality in patients with IgA nephropathy. Am J Kidney Dis. 2013;62:883-890. 
Clinical Epidemiology is an international, peer-reviewed, open access, online journal focusing on disease and drug epidemiology, identification of risk factors and screening procedures to develop optimal preventative initiatives and programs. Specific topics include: diagnosis, prognosis, treatment, screening, prevention, risk factor modification,

Submit your manuscript here: https://www.dovepress.com/clinical-epidemiology-journal
Dovepress

systematic reviews, risk and safety of medical interventions, epidemiology and biostatistical methods, and evaluation of guidelines, translational medicine, health policies and economic evaluations. The manuscript management system is completely online and includes a very quick and fair peer-review system, which is all easy to use. 